\title{
USO DE PARCELAS DE AREIA PARA O MONITORAMENTO DE IMPACTO DE ESTRADAS SOBRE A RIQUEZA DE ESPÉCIES DE MAMÍFEROS ${ }^{1}$
}

\author{
Leandro Moraes Scoss², Paulo de Marco Júnior², Elias Silva³e Sebastião Venâncio Martins ${ }^{3}$
}

\begin{abstract}
RESUMO - O monitoramento de fauna é um procedimento essencial na determinação da eficiência de uma unidade de conservação. Os objetivos deste artigo foram avaliar o uso de parcelas de areia para registro de pegadas de mamíferos, para estimar a riqueza de espécies que utilizam habitats cortados por estradas, e analisar a aplicabilidade do método em um programa de monitoramento. Os levantamentos foram conduzidos no Parque Estadual do Rio Doce-MG, através do uso de duas grades de amostragem compostas por três transectos paralelos, a diferentes distâncias da estrada (12,82 e $152 \mathrm{~m}$ ), monitorados durante os meses de março a novembro de 2000 . As estimativas de riqueza de espécie foram obtidas pelo programa EstimateS, e o programa MONITOR foi utilizado para calcular o poder da análise para estimar uma probabilidade de detecção $(\mathrm{p}=0,90)$ de um declínio $(5 \%)$ do número de espécies às margens da estrada. As estimativas de riqueza de espécies para os transectos variaram entre 5,99 (DP 0,99) e 15,07 (DP 1,75). Os resultados indicam que um programa de monitoramento precisaria de três levantamentos por ano, durante quatro anos consecutivos, utilizando o mesmo protocolo de amostragem para detectar um declínio (5\%) no número de espécies que utilizam a estrada, com probabilidade de 0,90. O uso de parcelas de areia é um método de fácil aplicação, que se mostrou adequado para estimar a riqueza de espécies de mamíferos, podendo contribuir com informações e previsões para o manejo e a conservação destas espécies.
\end{abstract}

Palavras-chave: Mamíferos, monitoramento e parcelas de areia.

\section{SAND-PLOT USE FOR MONITORING ROAD IMPACT ON MAMMAL SPECIES RICHNESS}

\begin{abstract}
Faunal monitoring is an essential procedure to evaluate the efficiency of a conservation unit. The aims of this paper were to evaluate the use of sand-plots to record mammal tracks for estimating species richness in habitats crossed by roads and to analyze the applicability of this method to propose a monitoring program. The survey was carried out in Rio Doce State Park, $M G$, in two sampling grids composed by 3 parallel transects at different distances from the forest (12, 82 and $152 \mathrm{~m}$ ), monitored from March to November 2000. Species richness estimates were obtained by applying the EstimateS program and the MONITOR program to calculate the power of the analysis to estimate a probability of detection $(p=0.90)$ of a decline $(5 \%)$ in the number of species on the road borders. Species richness estimates in the transects varied between 5.99 (SD 0.99) and 15.07 (SD 1.75). The results indicate that a monitoring program needs at least 3 surveys per year during 4 consecutive years using the same sampling protocol to detect a 5\% decline in species richness on the road, with 0.90 probability. The use of sand plots is an easy-to use method that can be applied to estimate mammal species richness, and provide important information to management and conservation of these species.
\end{abstract}

Key words: $\quad$ Mammals, monitoring program, sand plots

Recebido para publicação em 24.4.2002 e aceito para publicação em 17.2.2004.

2 Laboratório de Ecologia Quantitativa, Departamento de Biologia Geral da Universidade Federal de Viçosa - UFV, $36570-000$ Viçosa-MG, <lmscoss@ univale.br>; ${ }^{3}$ Departamento de Engenharia Florestal da UFV. 


\section{INTRODUÇÃO}

Estradas são vitais para o crescimento da economia de uma nação. Geram novas oportunidades de serviços e empregos e a instalação de novos pontos residenciais e industriais, o que resulta na atração de pessoas para áreas antes não habitadas (Fearnside, 1989, 1990; Wilkie et al., 2000). Muitas destas novas áreas ocupadas por estradas e, conseqüentemente, urbanizadas são ecologicamente vulneráveis ou apresentam alto risco de perda da integridade biótica das comunidades que compõem a paisagem (Karr, 1993). Associada a toda paisagem que recebe estradas, está a ocorrência de impactos negativos sobre a integridade biótica, tanto de ecossistemas terrestres como aquáticos (Trombulak \& Frissel, 2000).

Uma vez que a estrada subdivide uma paisagem natural, removendo uma porção de habitat, ela inibe a dispersão e migração de espécies e facilita a propagação de distúrbios (por exemplo fogo, poluentes e caça) para o interior dessas áreas. De acordo com Schonewald-Cox \& Buechner (1992), a fragmentação de unidades de conservação por estradas afeta negativamente as espécies que: i) não se dão bem em habitats de borda, ii) são sensíveis ao contato humano, iii) ocorrem em baixas densidades, iv) são improváveis ou incapazes de atravessar estradas e v) procuram estradas para se aquecer ou se alimentar. Os autores sugerem que estradas podem atuar tanto como barreiras, como corredores, ou ambos.

A baixa densidade local de muitas espécies de mamíferos e o tamanho de suas áreas de vida, aliados ao hábito noturno, dificultam a realização de estudos de determinação da composição, estrutura e dinâmica dessas populações. $\mathrm{O}$ emprego de indicadores indiretos da presença de mamíferos é mais barato, rápido e de mais fácil observação no campo, comparados com os métodos diretos (observação e captura). Os índices indiretos são baseados na contagem de rastros (Van Dyke et al., 1986; Wilson et al., 1996; Scoss \& De Marco, 2000a), vocalizações ou outros sons, visitas a estações de cheiro (Roughton \& Sweeny, 1982; Conner et al., 1983; Thompson et al., 1989), ossadas e fezes (Eisenberg et al., 1970) e tamanhos de área de vida (Schaller \& Crawshaw, 1980; Rodrigues \& Monteiro-Filho, 2000). Em geral, assume-se que os índices indiretos sejam positivos e apresentam, preferencialmente, relação linear com a abundância real das populações (Wilson et al., 1996).

No entanto, além das questões práticas do uso destes métodos na estimativa de mudanças dentro de comunidades naturais, existe um crescente interesse de melhorar a capacidade de detecção de mudanças através da construção de hipóteses e testes estatísticos formais destas mudanças (Caughley \& Gunn, 1996). Uma das preocupações mais recorrentes no uso de testes de hipóteses em Ecologia, particularmente em Biologia da Conservação, é a estimativa do poder destes testes (Gerrodette, 1987; Taylor \& Gerrodette, 1993; Osenberg et al., 1994; Hayes \& Steidl, 1997; Thompson et al., 2000). O poder de um teste é definido como a probabilidade de rejeitar uma hipótese, sendo ela falsa (Zar, 1999).

Este tipo de preocupação é crucial em Biologia da Conservação, porque pequenos tamanhos amostrais são comuns no estudo de populações raras que vivem em sistemas fragmentados (por exemplo, o Projeto Dinâmica Biológica de Fragmentos Florestais - PDBFF, na Amazônia brasileira). Nestes casos é muito importante avaliar o poder desses testes para verificar o alcance de nossas conclusões, bem como para permitir um planejamento mais apropriado que garanta que o esforço de coleta permita que resultados conclusivos sejam atingidos.

Uma das questões mais importantes nesse contexto é se uma unidade de conservação é eficiente na manutenção da biodiversidade. Se realmente é, a riqueza de espécies deveria ser estável (equilíbrio dinâmico) a ponto de garantir a integridade biótica dessas áreas (Karr, 1993).

Com base no exposto, o objetivo deste trabalho foi avaliar o uso do método de parcelas de areia para registro de pegadas de mamíferos no Parque Estadual do Rio Doce (PERD), visando estimar a riqueza de espécies que utilizam habitats cortados por estradas, bem como analisar a aplicabilidade do método e do protocolo de amostragem para um programa de monitoramento da riqueza de espécies de mamíferos às margens de estradas.

\section{MATERIAL E MÉTODOS}

O levantamento foi conduzido no Parque Estadual do Rio Doce (PERD), maior remanescente de Floresta Atlântica de Minas Gerais. O PERD possui uma área de aproximadamente 36.000 ha, e está localizado na porção sudeste do Estado. A altitude varia entre 230 e $515 \mathrm{~m}$, e o clima é tropical quente semi-úmido, com uma estação chuvosa no verão e seca de quatro a cinco meses no inverno (Nimer, 1989). Seu entorno faz divisa com os municípios de Marliéria, Timóteo e Dionísio. A área do 
parque é essencialmente coberta por uma floresta tropical úmida, entre os meridianos $42^{\circ} 38^{\prime} \mathrm{O}$ e $48^{\circ} 28^{\prime} \mathrm{O}$, e os paralelos 1941' S e 19³0' S (Godinho, 1996), e pode ser classificada como semidecídua tropical (Gilhuis, 1986). A pluviosidade média anual no parque é de $1.480,3 \mathrm{~mm}$, a temperatura média anual de $21,9^{\circ} \mathrm{C}$ e $\mathrm{o}$ período de déficit hídrico de maio a setembro (Gilhuis, 1986).

O PERD possui uma estrada interna (estrada da Ponte Queimada) que o atravessa em $22 \mathrm{~km}$, desde o limite leste até o oeste. A maior parte da estrada corta uma área coberta por mata primária que apresenta alto grau de riqueza de habitat faunístico (Magro, 1988). Nesta estrada o fluxo de veículos é menor que 50 veículos/ dia, caracterizando-se pelo trânsito de turistas e moradores dos municípios adjacentes.

O protocolo de amostragem foi composto por duas grades de $160 \times 200 \mathrm{~m}$ (3,2 ha cada). Cada grade tinha três transectos paralelos à estrada, espaçados $70 \mathrm{~m}$ um do outro, no sentido do interior da mata. Para cada transecto foram distribuídas 20 parcelas de areia, espaçadas em $10 \mathrm{~m}$. As dimensões das parcelas foram de 0,50x0,50 m, preenchidas com areia fina $(2-4 \mathrm{~cm}$ de altura). No período de coleta, entre março e novembro de 2000 (média de dois dias/mês), as parcelas foram limpas, molhadas e iscadas com banana um dia antes da coleta. Após permanecerem 24 horas iscadas, cada parcela foi percorrida, verificando-se a presença ou não de pegadas de mamíferos, identificando-as com o auxílio de guias de campo (Becker \& Dalponte, 1991; Emmons, 1997), anotando a espécie, o local/estação, a data e o horário e, finalmente, iscando novamente as parcelas. Sempre que necessário, as parcelas foram molhadas entre um dia de coleta e outro.

A proporção de pegadas de cada espécie nas parcelas de areia foi utilizada como indicativo de sua abundância relativa. Para estimar a riqueza de espécies total para cada transecto, utilizou-se o programa EstimateS versão 6.0b1 (Colwell, 2000), e foram consideradas apenas as estimativas geradas pelo procedimento Jackknife (Heltshe \& Forrester, 1983).

Utilizou-se o programa MONITOR (Gibbs, 1995) para calcular o poder das estimativas de riqueza de espécies, obtido pelo procedimento JACKKNIFE. Para realizar esta análise houve necessidade de saber o número de espécies esperadas para cada transecto e sua variação (desvio-padrão, DP). A frequiência de registro de pegadas de cada espécie nas parcelas de areia, nos seis transectos amostrados, foi utilizada para estimar a riqueza de espécies e para o cálculo da variabilidade de cada estimativa. As simulações foram feitas com um teste bicaudal, a fim de estimar o esforço necessário para alcançar um poder de rejeição da hipótese nula de $90 \%$, para identificar um declínio no número de espécies que utilizam as margens da estrada da Ponte Queimada/PERD. Para tanto, manteve-se o número de transectos constante $(\mathrm{n}=6)$ e duas variáveis foram simuladas: 1) o número de vezes que cada transecto deve ser repetido por ano; e 2) o número de anos de monitoramento necessários para detectar o declínio.

\section{RESULTADOS E DISCUSSÃO}

Em um total de 1.200 parcelas de areia, foram registradas 16 espécies de mamíferos de médio e grande porte. Aproximadamente $87 \%$ do total de parcelas apresentou pelo menos um registro de pegada de mamíferos durante o período de coleta. Dos mamíferos registrados no parque e passíveis de identificação por pegadas (Artiodactyla, Perissodactyla, Carnivora, Edentata, Lagomorpha, parte de Rodentia e parte de Marsupialia), foram registrados $66,67 \%$ dos mamíferos listados para o PERD (Stallings et al., 1991). As espécies mais abundantes foram Dasyprocta sp. (cutia), Didelphis spp. (gambá), Sylvilagus brasiliensis Linnaeus, 1758 (tapiti) e Cuniculus paca Brisson, 1762 (paca), que apresentaram, respectivamente, os seguintes índices de abundância ( $\mathrm{n}^{\mathrm{o}}$ de pegadas/parcelas de areia): 0,77, 0,11, 0,09 e 0,06 .

As estimativas de riqueza de espécies para cada transecto variaram entre 5,99 (DP 0,99) e 15,07 (DP 1,75). Entre as espécies ameaçadas, foram identificadas a onçaparda (Puma concolor Linnaeus, 1771), o gato-maracajá (Leopardus wiedii Schinz, 1821) e a anta (Tapirus terrestris Linnaeus, 1758), que estão presentes no Livro Vermelho de Espécies Ameaçadas de Extinção da Fauna de Minas Gerais, como espécies criticamente em perigo ou ameaçadas (Machado et al., 1998). As demais espécies (Mazama americana Erxleben, 1777, Cebus apella Linnaeus, 1758, Nasua nasua Linnaeus, 1766, Cerdocyon thous Linnaeus, 1766, Eira barbara Linnaeus, 1758, Sciurus aestuans Linnaeus, 1766, Dasypus novemcinctus Linnaeus, 1758, Galictis spp. e Conepatus semistriatus Boddaert, 1784) foram registradas com baixa frequiência, principalmente os carnívoros, não permitindo o cálculo de índices de abundância confiáveis.

R. Árvore, Viçosa-MG, v.28, n.1, p.121-127, 2004 
As estimativas de riqueza de espécies de mamíferos através do método das parcelas de areia distribuídas em transectos no interior da mata do PERD foram decisivas na constatação de que muitas espécies utilizam a estrada do parque e que seguramente outras, não-registradas neste estudo, também a utilizam, como Procyon cancrivorus (mão-pelada), Panthera onca (onça-pintada) e Tayassu tajacu e T. pecari (porcos-do-mato).

A diferença entre as estimativas, entre os transectos, indica que há espécies exclusivas que utilizam determinados transectos e outros não, aumentando a variação entre eles. O poder do teste fornecido pelo MONITOR, para avaliar o protocolo de amostragem proposto, apresentou probabilidade de 0,99 , indicando que tanto o número de transectos como o número de réplicas para cada transecto por levantamento (dois dias) foram suficientes para estimar o número de espécies que utilizam as margens da estrada da Ponte Queimada/PERD. Este levantamento inicial da riqueza de espécies apresentou um poder de análise satisfatório, e a partir dessas informações foi simulado um programa de monitoramento de espécies de mamíferos para a estrada interna ao PERD.

As simulações realizadas para detectar 5\% de declínio da estimativa da riqueza de espécies de mamíferos, com probabilidade de detecção de 0,90 , apresentaram duas situações distintas de monitoramento (Quadro 1). A primeira indica dois levantamentos por ano, utilizando o mesmo protocolo de amostragem, por cinco anos consecutivos. Já a segunda opção seria realizar três levantamentos por ano, durante quatro anos consecutivos, para atingir os mesmos objetivos.

Entre os dois cenários possíveis para monitorar a riqueza de espécies de mamíferos na estrada do PERD, optou-se pelo segundo, onde são necessários três levantamentos por ano, ao longo de quatro anos, para detectar $5 \%$ de declínio. Por se tratar de impactos provocados por estradas, preferiu-se adotar a opção mais conservadora, o que permite tomar decisões mais rápidas, principalmente em relação às espécies ameaçadas de extinção e, sobretudo, as exóticas, que podem estar invadindo o parque, utilizando a estrada como corredor de dispersão (Shonewald-Cox \& Buechner, 1992). Os resultados do poder do teste indicam que é possível propor um programa adequado de monitoramento, utilizando parcelas de areia distribuídas em transectos no interior da mata, para detectar declínio no número de espécies de mamíferos que utilizam estradas.

R. Árvore, Viçosa-MG, v.28, n.1, p.121-127, 2004
Quadro 1 - Probabilidade de detecção de declínio do número de espécies de mamíferos terrestres, às margens da estrada da Ponte Queimada/PERD/MG, utilizando o método de registro de pegadas em parcelas de areia. $\mathrm{Pd}$. Porcentagem de declínio; e $\mathrm{N}=$ número de levantamentos por ano

Table 1 - Probability of detecting declines in terrestrial mammal species richness, at Ponte Queimada/PERD/ MG road borders, using sand-plot technique in transects in the forest; Pd. Percent decline; N. Number of surveys per year

\begin{tabular}{|c|c|c|c|c|c|}
\hline \multirow{2}{*}{ Período } & \multirow{2}{*}{$\mathrm{Pd}$} & \multicolumn{4}{|c|}{$\mathrm{N}$} \\
\hline & & 1 & 2 & 3 & 4 \\
\hline \multirow{5}{*}{3 anos } & 1 & 0,07 & 0,06 & 0,14 & 0,12 \\
\hline & 3 & 0,19 & 0,18 & 0,26 & 0,16 \\
\hline & 5 & 0,25 & 0,46 & 0,68 & 0,76 \\
\hline & 7 & 0,50 & 0,74 & 0,85 & 0,96 \\
\hline & 9 & 0,58 & 0,91 & 0,99 & 0,98 \\
\hline \multirow{5}{*}{4 anos } & 1 & 0,04 & 0,13 & 0,12 & 0,16 \\
\hline & 3 & 0,22 & 0,49 & 0,60 & 0,44 \\
\hline & 5 & 0,47 & 0,83 & 0,93 & 0,94 \\
\hline & 7 & $\begin{array}{l}0,82 \\
0,82\end{array}$ & 0,97 & 1,00 & $\begin{array}{l}1,00 \\
. \\
3\end{array}$ \\
\hline & 9 & 0,97 & 1,00 & 1,00 & 1,00 \\
\hline \multirow{5}{*}{5 anos } & 1 & 0,08 & 0,15 & 0,20 & 0,26 \\
\hline & 3 & 0,38 & 0,70 & 0,87 & 0,67 \\
\hline & 5 & 0,86 & 0,98 & 1,00 & 1,00 \\
\hline & 7 & 0,96 & 1,00 & 1,00 & 1,00 \\
\hline & 9 & 1,00 & 1,00 & 1,00 & 1,00 \\
\hline
\end{tabular}

A precisão de uma estimativa de índice de abundância depende da consistência e da rígida padronização da técnica de amostragem, porque delas depende a variância dos índices obtidos. Considerando a complexidade de se trabalhar com muitas espécies ao mesmo tempo (Conner et al., 1983), além do fato de que um mesmo protocolo de amostragem provavelmente não é relevante para gerar índices de abundância viáveis para todas elas, preferiu-se discutir especificamente as estimativas de riqueza de espécie. Questões como distância entre parcelas de areia, independência entre parcelas (espacial e temporal) e esforço amostral podem tornar o protocolo inadequado para algumas espécies, principalmente em estudos que consideram mais de uma espécie ou grupo ecológico (Roughton \& Sweeny, 1982; Conner et al., 1983; Scoss \& De Marco, 2000b). Entretanto, apesar de o método das parcelas de areia não ser perfeito, ele é eficiente porque pode ser aplicado em grandes áreas, é facilmente padronizado e pode fornecer informações importantes sobre o uso de habitats (Nottingam et al., 1989). 
A partir dos resultados deste trabalho, sugere-se que este mesmo protocolo também seja utilizado em todos os empreendimentos nos quais o impacto de estradas seja avaliado. Os estudos e seus respectivos relatórios de impactos ambientais (EIA/RIMA), se mantiverem este tipo de protocolo, devem dar ênfase ao monitoramento como estratégia de avaliar problemas futuros de manutenção da integridade biótica dos sistemas. Os modelos indicam que os efeitos decorrentes da fragmentação dos habitats induzem mudanças que podem surgir anos depois do estabelecimento do impacto (Terborgh, 1988; Dirzo \& Miranda, 1990; Saunders et al., 1991; Laurance, 1991, 1997; Murcia, 1995; Kapos et al., 1997; Vianna et al., 1997), reforçando a importância dos estudos de monitoramento e do estabelecimento de um protocolo de amostragem prévio, para o sucesso da identificação dos impactos gerados por estradas.

\section{CONCLUSÕES}

As técnicas de monitoramento de populações devem ser testadas adequadamente antes da sua aplicação em larga escala. Contudo, o método das parcelas de areia distribuídas em transectos no interior da mata para registro de pegadas de mamíferos mostrou-se um método eficiente para estimar a riqueza de espécies que utilizam a estrada interna ao PERD. Este método pode ser útil para estudos de impacto e diagnose ambiental, plano de manejo e gestão de unidades de conservação, gerando informações e previsões importantes para o manejo e a conservação de mamíferos, especialmente os ameaçados de extinção.

\section{AGRADECIMENTO}

A Fundação O Boticário de Proteção à Natureza, The John D. and Catherine T. MacArthur Foundation, Instituto de Pesquisas da Mata Atlântica (IPEMA) e Coordenação de Aperfeiçoamento de Pessoal do Ensino Superior (CAPES), pelo apoio financeiro. Ao Instituto Estadual de Florestas de Minas Gerais (IEF/MG), por conceder as devidas licenças de coleta para realizar este estudo no Parque Estadual do Rio Doce e pela concessão dos alojamentos durante as campanhas de campo.

\section{REFERÊNCIAS BIBLIOGRÁFICAS}

BECKER, M.; DALPONTE, C. J. Rastros de mamíferos silvestres brasileiros: um guia de campo. Brasília: Universidade de Brasília, 1991. 181 p.
CAUGHLEY, G.; GUNN, A. Conservation Biology in theory and practice. Massachusetts: Blackwell Science, 1996. $459 \mathrm{p}$.

COLWELL, R. K. ESTIMATE S (version 6.0 b1.) Statistical estimation of species richness and shared species from samples, 2000. University of Connecticut. Disponível em: http://viceroy.eeb.uconn.edu/estimates. Acesso em: 18 jul. 2001.

CONNER, M. C.; LABISKY, R. F.; PROGULSKE, D. R. JR. Scent-station indices as measures of population abundance for Bobcats, Raccoons, Gray Foxes, and Opossums. Wildlife Society Bulletin, v. 11, n. 2, p. 146152, 1983.

DIRZO, R.; MIRANDA, A. Contemporary neotropical defaunation and the forest structure, function, and diversity - a sequel to John Terborgh. Conservation Biology, v. 4, p. 444-447, 1990.

EISENBERG, J. F.; SANTIAPILLAI, C.; LOCKHART, M. The study of wildlife populations by indirect methods. The Ceylon J. of Science, Biology Sciences, v. 8, n. 2, p. 53-62, 1970.

EMMONS, L. H. Neotropical rainforest mammals: a field guide. 2.ed. Chicago: University of Chicago Press, 1997. 307 p.

FEARNSIDE, P. M. A ocupação humana de Rondônia: impactos, limites e planejamento. Brasília: Programa Polonoroeste, 1989. $76 \mathrm{p}$.

FEARNSIDE, P. M. Rondônia: estradas que levam à devastação. Ciência Hoje, v. 11, n. 61, p. 47-52, 1990.

GERRODETTE, T. A power analysis for detecting trends. Ecology, v. 68, n. 5, p. 1364-1372, 1987.

GIBBS, J. P. Monitor users manual. New Haven: Yale University, 1995.

GODINHO, A. L. Peixes do Parque Estadual do Rio Doce. Belo Horizonte: Instituto Estadual de Florestas / Universidade Federal de Minas Gerais, 1996. v. 1. 48 p.

GULHUIS, J. P. Vegetation Survey on the Parque Florestal Estadual do Rio Doce - MG - Brazil. 1986. 86 f. Dissertação (Mestrado em Ciência Florestal) Agricultural University of Wageningen, Wageningen, 1986.

R. Árvore, Viçosa-MG, v.28, n.1, p.121-127, 2004 
HAYES, J. P.; STEIDL, R. J. Statistical power analysis and amphibian population trends. Conservation Biology, v. 11, n. 1, p. 273-275, 1997.

HELTSHE, J. F.; FORRESTER, N. E. Estimating species richness using the JACKKNIFE procedure. Biometrics, v. 39, p. 1-11, 1983.

KAPOS, V. et al. Edge-related changes in environmental and plant responses due to forest fragmentation in central Amazonia. In: LAURANCE, W. F.; BIERREGARD JR., R. O. (Eds.). Tropical forest remnants: ecology, management, and conservation of fragmented communities. Chicago: University of Chicago Press, 1997. p. 33-44.

KARR, J. R. Measuring biological integrity: lessons from streams. In: WOODLEY, S.; KAY, J.; FRANCIS, G. (Eds.). Ecological integrity and the management of ecosystems. London: St. Lucie Press, 1993. p. 83-104.

LAURANCE, W. F. Edge effects in tropical forest fragments: application of a model for the design of nature reserves.

Biological Conservation, v. 57, p. 205-219, 1991.

LAURANCE, W. F. Hyper-disturbed parks: Edge effects and the ecology of isolated rainforests reserves in tropical Australia. In: LAURANCE, W. F.; BIERREGARD JR., R. O. (Eds.) Tropical forest remnants: ecology, management, and conservation of fragmented communities. Chicago: University of Chicago Press, 1997. p. 71-83.

MACHADO, A. B. M. et al. Livro vermelho das espécies ameaçadas de extinção da fauna de Minas Gerais. Belo Horizonte: Fundação Biodiversitas, 1998. 608 p.

MAGRO, T. C. Avaliação da qualidade de habitat faunístico pela análise de bordas. 1988. $95 \mathrm{f}$. Dissertação (Mestrado em Ciência Florestal) - Universidade Federal de Viçosa, Viçosa, 1988.

MURCIA, C. Edge effects in fragmented forests: implications for conservation. Trends Ecology Evolution, v. 10, p. 58-62, 1995.

NIMER, E. O modelo de zonas de vida de Holdridge; conceito e procedimentos metodológicos fundamentais. Cadernos de Geociencia). Rio de Janeiro: 1989. p. 33-44.

NOTTINGHAM Jr., B. G.; JOHSON, K. G.; PELTON, M. R. Evaluation of scent-station surveys to monitor raccoon density. Wildlife Society Bulletin, v. 17, p. 29-35, 1989.

R. Árvore, Viçosa-MG, v.28, n.1, p.121-127, 2004
OSENBERG, C. W. et al. Detection of environmental impacts - natural variability, effect size, and power analysis. Ecology Applied, v. 4, n. 1, p. 16-30, 1994.

RODRIGUES, F. H. G.; MONTEIRO-FILHO, E. L. A. Home range and activity patterns of pampas deer in Emas National Park, Brazil. Journal of Mammalogy, v. 81, n. 4, p. 1136-1142, 2000.

ROUGHTON, R. D.; SWEENY, M. W. Refinements in scent-station methodology for assessing trends in carnivore populations. Journal Wildl. Management, v. 46, n. 1, p. 217-229, 1982.

SAUNDERS, D. A.; HOOBS, R. J.; MARGULES, C. R. Biological consequences of ecosystem fragmentation: a review. Conservation Biology, v. 5, p. 18-32, 1991.

SCHALLER, G. B.; CRAWSHAW JR., P. G. Movement patterns of jaguar. Biotropica, v. 12, n. 3, p. 161-168, 1980.

SCHONEWALD-COX, C.; BUECHNER, M. Park protection and public roads. In: FIELDER, P. L.; JAIN, S. K. (Eds.). Conservation Biology - The Theory and practice of nature conservation, preservation and management. London: Chapman and Hall, 1992. p. 375-395.

SCOSS, L. M.; DE MARCO Jr., P. Avaliação metodológica do uso de pegadas de mamíferos em estudos de biodiversidade. In: CONGRESSO E EXPOSIÇÃO INTERNACIONAL SOBRE FLORESTAS - FOREST, 6 , 2000, Rio de Janeiro. Anais... Rio de Janeiro: Instituto Ambiental Biosfera, 2000b. p. 457-459.

SCOSS, L. M.; DE MARCO Jr., P. Estradas no parque: efeitos da fragmentação interna sobre a intensidade de uso do habitat por mamíferos terrestres. In: CONGRESSO BRASILEIRO DE UNIDADES DE CONSERVAÇÃO, 2., 2000, Campo Grande. Anais... Campo Grande: Rede Nacional Pró-Unidades de Conservação: Fundação O Boticário de Proteção à Natureza, 2000a. p. 770-776.

STALLINGS, J. R. et al. Mamíferos do Parque Estadual do Rio Doce, Minas Gerais, Brasil. Revista Brasileira de Zoologia, v. 7, n. 4, p. 663-677, 1991.

TAYLOR, B. L.; GERRODETTE, T. The uses of statistical power in conservation biology: The vaquita and northern spotted owl. Conservation Biology, v. 7, n. 3, p. 489-500, 1993.

TERBORGH, J. The big things that run the world - a sequel to E. O. Wilson. Conservation Biology, v. 2, p. 402-403, 1988. 
THOMPSON, I. D. et al. Use of track transects to measure the relative occurence of some boreal mammals in uncut forest and regeneration stands. Cannadian Journal of Zoology, v. 67, p. 1816-1823, 1989.

THOMPSON, P. M. et al. Combining power analysis and population viability analysis to compare traditional and precautionary approaches to conservation of coastal cetaceans. Conservation Biology, v. 14, n. 5, p. 1253 $1263,2000$.

TROMBULAK, C. S.; FRISSEL, A. C. Review of ecological effects of roads on terrestrial and aquatic communities. Conservation Biology, v. 14, n. 1, p. 18-30, 2000 .

VAN DYKE, F. G.; BROCKE, R. H.; SHAW, H. G. Use of road track counts as indices of Mountain Lion Presence.

Journal Wildl. Management, v. 50, n. 1, p. 102-109, 1986.
VIANA, V. M.; TABANEZ, A. A. J.; BATISTA, J. L. F. Dynamics and restoration of forest fragments in the Brazilian Atlantic moist forest. In: LAURANCE, W. F.; BIERREGARD JR., R. O. (Eds.) Tropical forest remnants: ecology, management, and conservation of fragmented communities. Chicago: University of Chicago Press, 1997. p. 351-365.

WILKIE, D. et al. Roads, development, and conservation in the Congo basin. Conservation Biology, v. 14, n. 6 , p. 1614-1622, 2000.

WILSON, D. E. et al. Measuring and monitoring biological diversity: standard methods for mammals. Washington: Smithsonian Press, 1996. 409 p.

ZAR, J. H. Biostatistical analysis. Englewood Cliffs, Prentice-Hall, 1999. 929 p. 